\section{Chloroquine and Hydrox- ychloroquine in COVID-19: Challenges and the Need for Caution in Low-Resource Settings}

Sir,

Chloroquine (anti-malarial) and hydroxychloroquine (immunosuppressant) have been heavily discussed as novel therapeutic agents in the treatment of COVID-19. ${ }^{1}$ In fact, in low-income regions, chloroquine may represent the only feasible treatment option that directly targets the disease process. Consequently, increased demand for the agent has led to stockpiling in households (in many cases, without prescription) and shortages in the market. However, a broader view of public health underscores the dangers of indiscriminate chloroquine use,especially in lowresource countries.

In general, the toxicity profiles of the aforementioned medicines have been extensively observed and documented; however, chloroquine and hydroxychloroquine have seldom been tested in critically-ill patients with COVID-19. Unfortunately, this patient-population is the most likely to require added treatment; however, available clinical trials do not describe if they can tolerate the same doses as otherwise healthy patients - that is, the doses they need to significantly improve theircondition.

Secondly, known development of artemisinin resistance and recent evidence of $100 \%$ efficacy of chloroquine in eradicating uncomplicated Plasmodium vivax malaria in Pakistan supports the continued use of chloroquine as anti-malarial therapy, ${ }^{2}$ as per WHO guidelines. In areas with endemicity of chloroquine-sensitive malaria, overuse of chloroquine in the COVID-19 crisis may precipitate not only supply deficiency; but also the spread of chloroquine resistance, which has the potential to restrict effective therapeutic options for malaria as a longterm consequence.

Lastly, given fluid discharge standards for COVID-19 in the event of discharged patients re-testing positive, ${ }^{3}$ the duration of chloroquine/hydroxychloroquine use in the hospital setting (and potentially after discharge) would also need re-evaluation in the context of availability per patient and cumulative toxicity. If chloroquine and hydroxychloroquine were to be discontinued following a negative PCR and the given patients were to re-test positive in the future, it may become necessary to consider the possibility of reduced effectiveness of the same agent in second-line treatment due to antiviral resistance. The idea of drug resistance has been explored previously with MERS-CoV (an older species of coronavirus), with experimental studies having suggested a genetic basis for resistance to drugs such as amantadine and interferons. ${ }^{4,5}$ Therefore, it may be reasonable to predict similar challenges in the clinical course of COVID-19 with prolonged/repetitive antiviral therapy.

In conclusion, certain limitations of the low-resource environment necessitate judicious use of chloroquine/hydroxychloroquine. Even in the midst of a pandemic, panoramic consideration of how healthcare practices may affect the epidemiology of existing endemic diseases is crucial.

\section{CONFLICT OF INTEREST:}

Author declared no conflict of interest.

\section{AUTHOR'S CONTRIBUTION:}

Conception of work, drafting and revision, final approval and accountability for all aspects of the work.

\section{REFERENCES}

1. Gao J, Tian Z, Yang X. Breakthrough: Chloroquine phosphate has shown apparent efficacy in treatment of COVID-19 associated pneumonia in clinical studies. Biosci Trends 2020; 14(1):72-3.

2. Waqar T, Khushdil A, Haque K. Efficacy of chloroquine as a first line agent in the treatment of uncomplicated malaria due to plasmodium vivax in children and treatment practices in Pakistan: A pilot study. J Pak Med Assoc 2016; 66(1):30-3.

3. Zhang JF, Yan K, Ye HH, Lin J, Zheng JJ, Cai T. SARS-CoV-2 turned positive in a discharged patient with COVID-19 arouses concern regarding the present standard for discharge. Int J Infect Dis 2020; S1201-9712(20)30126-0.

4. Chan JF, Chan KH, Kao RY, Kelvin KW To, Zheng BJ, Chen H, et al. Broad-spectrum antivirals for the emerging Middle East respiratory syndrome coronavirus. J Infect 2013; 67(6):606-16.

5. Menachery VD, Gralinski LE, Mitchell HD, Dinnon KH, Leist $\mathrm{SR}, \mathrm{Graham} \mathrm{RL}$, et al. Middle-east respiratory syndrome coronavirus nonstructural protein 16 is necessary for interferon resistance and viral pathogenesis. mSphere 2017; 2(6):pii:e00346-17.

Mahnoor Islam

Dow Medical College, Karachi, Pakistan

Correspondence to: Dr. Mahnoor Islam, Dow Medical College, Baba-e-Urdu Road, Karachi, Pakistan

E-mail: mahnoorislam95@gmail.com

Received: April 02, 2020; Revised: April 06, 2020;

Accepted: June 18, 2020

DOI: https://doi.org/10.29271/jcpsp.2020.JCPSPCR.CR78 